\title{
Realization of the Optimal Universal Quantum Entangler
}

\author{
Fabio Sciarrino*, Francesco De Martini* and Vladimir Bužek ${ }^{\dagger}$ \\ * Dipartimento di Fisica and Istituto Nazionale per la Fisica della \\ Materia, \\ Universita' di Roma "La Sapienza", Roma, 00185 Italy, \\ $\dagger$ Research Center for Quantum Information, Institute of Physics, \\ Slovak Academy of Sciences \\ Dúbravská cesta 9, 84511 Bratislava, Slovakia.
}

(November 11, 2018)

\begin{abstract}
We present the first experimental demonstration of the "optimal" and "universal" quantum entangling process involving qubits encoded in the polarization $(\vec{\pi})$ of single photons. The structure of the "quantum entangling machine" consists of the quantum injected optical parametric amplifier by which the contextual realization of the $1 \rightarrow 2$ universal quantum cloning and of the universal NOT (U-NOT) gate has also been achieved.
\end{abstract}

Typeset using REVTEX 
The two distinctive features of quantum kinematics are the quantum superposition principle and the quantum entanglement. Among many consequences of the statistical character of quantum kinematics, a complete determination of the unknown state of a quantum system can be attained only when a complete measurement (i.e. the measurement of the quorum of observables) is performed on an infinite ensemble of identically prepared quantum objects. The measurement on a finite ensemble results in an imperfect reconstruction of the quantum state $[1]$.

If we consider the physical world to be represented by states of quantum objects then it is obvious that the quantum information (QI) processing is fundamentally different from any processing on a classical level. One of the main differences is that, in general, given just one physical object carrying a specific quantum information this one cannot be determined. In addition many operations on individual quantum objects prepared in unknown quantum states cannot be performed perfectly. A renowned example of such a constraint is the impossibility of cloning (copying) an unknown quantum state $|\Psi\rangle[2]$, i.e. a universal machine realizing exactly the transformation $|\Psi\rangle|0\rangle \rightarrow|\Psi\rangle|\Psi\rangle$, being $|0\rangle$ a known state of the copier, cannot exist. On the other hand an approximate, i.e. optimal, universal quantum cloning machine has been theoretically proposed [3] and experimentally realized $[4,5]$. Another relevant example of an impossible task is the "flipping" of unknown qubits [6,7], i.e. the realization of a universal NOT-gate operation $|\Psi\rangle \rightarrow\left|\Psi^{\perp}\right\rangle$ being: $\left\langle\Psi \mid \Psi^{\perp}\right\rangle=0$. The impossibility of flipping an unknown qubit has several interesting consequences. For instance, it has been shown that encoding information about unknown spatial spin orientation into parallel and antiparallel pairs of qubits is different. Specifically, more information is contained in the antiparallel spins. This purely quantum mechanical (QM) effect is due to the entanglement that appears in the process of optimal measurement. However, in spite of this constraint an optimal universal NOT gate has indeed been proposed [6] and realized [8].

To pursue at a deeper level this most significant quantum-classical endeavor consider here the central role of state-entanglement in quantum mechanics. As it is well known 
this fundamental physical condition, pervasive of the entire QI domain, is the key ingredient of all quantum nonlocality tests involving either Bell inequalities or Hardy's "ladder proofs" $[9,10]$. Furthermore, it lies at the core of important QI protocols as quantum teleportation, dense coding, etc. Following the above reasonings, one may ask then again whether it is possible to realize exactly the map $|\Psi\rangle|\Phi\rangle \rightarrow(|\Psi\rangle|\Phi\rangle+|\Phi\rangle|\Psi\rangle)$ which implies the entanglement of two quantum systems initially prepared in two unknown states $|\Psi\rangle$ and $|\Phi\rangle$. Alternatively, the same question can be raised for the relevant map $|\Psi\rangle \rightarrow\left(|\Psi\rangle\left|\Psi^{\perp}\right\rangle+\left|\Psi^{\perp}\right\rangle|\Psi\rangle\right) / \sqrt{2} \equiv\left|\left\{\Psi, \Psi^{\perp}\right\}\right\rangle$ implying the "translation" of the information originally encoded in any unknown state $|\Psi\rangle$ into the corresponding entangled Bell state. This question has been addressed in Ref. [11] where it is shown that again the perfect entangling transformation is generally impossible but, once again an approximate universal entangling machine can be designed. In addition and most interestingly, it can be shown that any optimal universal quantum entangler, i.e. the one maximizing the average fidelity of success, is realized within a combined, simultaneous realization of the optimal universal quantum cloning and of the optimal universal spin-flipping processes. In the present work we report the first experimental demonstration of the "optimal" and "universal" quantum entangling process within such a complex conceptual and experimental framework.

Let us assume that QI is encoded in the polarization $(\vec{\pi})$ of single photons. The structure of the "quantum entangling machine" and, contextually, of the $N=1$ to $M=2$ universal quantum cloning machine and of the universal NOT (U-NOT) gate is the quantum injected optical parametric amplifier (QI-OPA) [5,12]. The action of this rather complex machine can be described by the covariant transformation [3]:

$$
|\Psi\rangle|\downarrow\rangle_{C}|\downarrow\rangle_{A C} \Longrightarrow \sqrt{2 / 3}|\Psi\rangle|\Psi\rangle\left|\Psi^{\perp}\right\rangle_{A C}-\sqrt{1 / 3}\left|\left\{\Psi, \Psi^{\perp}\right\}\right\rangle|\Psi\rangle_{A C}
$$

where the first (unknown) state vector $|\Psi\rangle$ in the left-hand side of the equation corresponds to the input, the second state vector describes the system on which the information will be copied ("blank" qubit), represented by the "cloning channel" (C), i.e. the injection mode $k_{1}$, while the third state vector, the "anticloning channel" (AC), represents the state of the 
machine. Precisely, the state of the machine is a qubit associated with the AC mode $k_{2}$. The blank qubit and the cloner are initially in the known ground state $|\downarrow\rangle$. At the output of the machine we find the completely symmetrized state $\left|\left\{\Psi, \Psi^{\perp}\right\}\right\rangle$ and two cloned qubits in the $\mathrm{C}$ channel: $\rho=2 / 3|\Psi \Psi\rangle\left\langle\Psi \Psi|+1 / 3|\left\{\Psi, \Psi^{\perp}\right\}\right\rangle\left\langle\left\{\Psi, \Psi^{\perp}\right\}\right|$. The density operator $\rho$ describes the best possible approximation of the perfect entangled state $\left|\left\{\Psi, \Psi^{\perp}\right\}\right\rangle$. The most attractive feature of this entangling machine is that the fidelity of its performance, i.e. the distance between the output and the ideally entangled-state, does not depend on the input state $|\Psi\rangle$ and takes the constant value $F=1 / 3$. The machine itself after the cloning transformation is in the state $\rho_{A C}=1 / 3\left|\Psi^{\perp}\right\rangle\left\langle\Psi^{\perp}\right|+1 / 3 \times \mathbf{I}$, where $\mathbf{I}$ is the unity operator. This last density operator is the best possible approximation of the spin-flip (U-NOT) operation permitted by the quantum mechanics.

The symmetrization process was experimentally realized in a $2 \times 2$ dimensional Hilbert space of photon polarization $(\vec{\pi})$ simultaneously with the realization of the linearized $N=1$, $M=2$ cloning process. Consider first the case of an input $\vec{\pi}$-encoded qubit $|\Psi\rangle_{\text {in }}$ associated with a single photon with wavelength $(\mathrm{wl}) \lambda$, injected on the input mode $k_{1}$ of the QI-OPA, the other input mode $k_{2}$ being in the vacuum state [12]. As for previous works, the photon was injected into the a nonlinear (NL) BBO ( $\beta$-barium-borate) $1.5 \mathrm{~mm}$ thick crystal slab, cut for Type II phase matching and excited by a sequence of UV mode-locked laser pulses having duration $\tau \approx 140 f$ sec and wl $\lambda_{p}$. The relevant modes of the NL 3-wave interaction driven by the UV pulses associated with mode $k_{p}$ were the two spatial modes with wave-vector (wv) $k_{i}, i=1,2$, each supporting the two horizontal $(H)$ and vertical $(V)$ linear- $\vec{\pi}$ 's of the interacting photons. The QIOPA was $\lambda$-degenerate, i.e. the interacting stimulated emitted photons had the same wl's $\lambda=\frac{1}{2} \lambda_{p}=795 \mathrm{~nm}$. The NL crystal orientation was set as to realize the insensitivity of the amplification quantum efficiency $(Q E)$ to any input state $|\Psi\rangle_{i n}$, i.e. the universality $(\mathrm{U})$ of the entangling machine. It is well known that this key property is assured by the squeezing hamiltonian [12]: $\widehat{H}_{i n t}=i \chi \hbar\left(\widehat{a}_{\Psi}^{\dagger} \widehat{b}_{\Psi \perp}^{\dagger}-\widehat{a}_{\Psi \perp}^{\dagger} \widehat{b}_{\Psi}^{\dagger}\right)+h . c .$. The field operators sets $\left\{\widehat{a}_{\Psi}^{\dagger}, \widehat{a}_{\Psi}\right\},\left\{\widehat{a}_{\Psi \perp}^{\dagger}, \widehat{a}_{\Psi \perp}\right\},\left\{\widehat{b}_{\Psi}^{\dagger}, \widehat{b}_{\Psi}\right\}$ and $\left\{\widehat{b}_{\Psi \perp}^{\dagger}, \widehat{b}_{\Psi \perp}\right\}$ refer to two mutually 
orthogonal $\vec{\pi}$-states, $|\Psi\rangle$ and $\left|\Psi^{\perp}\right\rangle$, realized on the two interacting spatial modes $k_{1}$ and $k_{2}$ acted upon by the $\widehat{a}$ and $\widehat{b}$ operators, respectively. The $S U(2)$ invariance of $\widehat{H}_{\text {int }}$ implied by the U-condition, i.e. the independence of the OPA "gain" $g \equiv \chi t$ to any unknown $\vec{\pi}$ - state of the injected qubit, $t$ being the interaction time, allows the use of the subscripts $\Psi$ and $\Psi^{\perp}$ in Eq.(1) [12].

The QIOPA apparatus adopted in the present work was arranged in the self-injected configuration shown in Figure 1. The UV pump beam, back-reflected by a spherical mirror $M_{p}$ with $100 \%$ reflectivity and $\mu$-adjustable position $\mathbf{Z}$, excited the NL crystal in both directions $-k_{p}$ and $k_{p}$, i.e. correspondingly oriented towards the right hand side and the 1.h.s. of Fig.1. A Spontaneous Parametric Down Conversion (SPDC) process excited by the $-k_{p}$ UV mode created singlet-states of photon polarization $(\vec{\pi})$. The photon of each SPDC pair emitted over $-k_{1}$ was back-reflected by a spherical mirror $M$ into the NL crystal and provided the $N=1$ quantum injection into the OPA excited by the UV beam associated with the back-reflected mode $k_{p}$. Because of the low pump intensity, the probability of the unwanted $N=2$ injection has been estimated to be $10^{-2}$ smaller than the one for $N=1$. The twin SPDC photon emitted over mode $-k_{2}$, selected by the devices (Wave-Plate + Polarizing Beam Splitter: $\left.W P_{T}+P B S_{T}\right)$ and detected by $D_{T}$, provided the "trigger" of the overall conditional experiment. The three fixed quartz plates $(Q)$ inserted on the modes $k_{1}, k_{2}$ and $-k_{2}$ provided the compensation for the unwanted walk-off effects due to the birefringence of the NL crystal. An additional walk-off compensation into the BBO crystal was provided by the $\lambda / 4 \mathrm{WP}$ exchanging on mode $-k_{1}$ the $|H\rangle$ and $|V\rangle \vec{\pi}-$ components of the injected photon. Because of the EPR non-locality of the emitted singlet, the $\vec{\pi}$-selection made on $-k_{2}$ implied deterministically the selection of the input state $|\Psi\rangle_{i n}$ on the injection mode $k_{1}$. All adopted photodetectors $(D)$ were equal SPCM-AQR14 Si-avalanche single photon units with $Q E^{\prime} s \cong 0.55$. One interference filter with bandwidth $\Delta \lambda=6 \mathrm{~nm}$ was placed in front of each $D$.

Since the U-condition of the apparatus was already tested in previous experiments $[4,5]$ 
we limited ourselves to inject only one polarization state on the input mode $k_{1}$, i.e. $|\Psi\rangle_{i n}=$ $|H\rangle=|1,0\rangle_{k_{1}} \otimes|0,0\rangle_{k_{2}}$ where $\widehat{a}_{\Psi}^{\dagger}|0,0\rangle_{k_{1}}=|1,0\rangle_{k_{1}}$ and $|m, n\rangle_{k_{1}}$ represents a product state with $m$ photons of the mode $k_{1}$ having the polarization $\Psi=H$, and $n$ photons having the polarization $\Psi^{\perp}=V$. Assume the input mode $k_{2}$ to be in the vacuum state. The initial $\vec{\pi}$-state evolves according the unitary operator $\widehat{\mathbf{U}} \equiv \exp \left(-i \widehat{H}_{i n t} t\right)$ :

$$
\widehat{\mathbf{U}}|\Psi\rangle_{i n} \simeq|1,0\rangle_{k 1} \otimes|0,0\rangle_{k 2}+g\left(\sqrt{2}|2,0\rangle_{k 1} \otimes|0,1\rangle_{k 2}-|1,1\rangle_{k 1} \otimes|1,0\rangle_{k 2}\right)
$$

The above linearization procedure representing the 1st-order approximation for the pure output state vector $|\Psi\rangle_{\text {out }}$ for $t>0$, i.e. the restriction to the simplest $1 \rightarrow 2$ cloning case, is justified here by the small experimental value of the gain: $g \approx 0.1[5]$. The first term in the expression $\propto g$ in Eq.(2) expresses the simultaneous emission on mode $k_{1}$ of the $1 \rightarrow 2$ cloned state $|2,0\rangle_{k_{1}}$ corresponding to the state $|\Psi \Psi\rangle$ expressed by the general theory and on mode $k_{2}$ of the flipped version of the input qubit realizing the quantum U-NOT gate [5]. The second term expresses the emission on mode $k_{1}$ of the symmetrized state $|1,1\rangle_{k_{1}}$ under the present investigation. The two photons emitted over the mode $k_{1}$ impinged on a balanced beamsplitter $\left(B S_{1}\right)$ that coupled the mode $k_{1}$ to the output modes $a$ and $b$. We restrict our analysis to the cases in which the two photons emerge from different output ports of the beamsplitter. The first term in $g$ in Eq.(2) hence leads to the following normalized output state

$$
|\Psi\rangle_{\text {out }}=\sqrt{\frac{2}{3}}|H\rangle_{a}|H\rangle_{b}|V\rangle_{k 2}-\frac{1}{\sqrt{6}}\left(|H\rangle_{a}|V\rangle_{b}+|V\rangle_{a}|H\rangle_{b}\right)|H\rangle_{k 2}
$$

This state was analyzed by the simultaneous excitation of the two detector pairs ( $a$ and $b$ ) coupled respectively by the two $\vec{\pi}$-analyzers $P B S_{a}$ and $P B S_{b}$ to the two output modes of the Beam Splitter $\left(B S_{1}\right)$, and of the detector pair (2) associated to the polarizing beam splitter $P B S_{2}$ (Fig. 1). The histogram shown in Fig. 2 reports the experimental realization of the output state $\propto g$ by expressing the probabilities of the various simultaneous state contributions in Eq.(3). The variable $X Y Z$ of the histogram reads as follows: $X=$ polarization $\vec{\pi}$-state detected by the detector pair $(a)$ on the mode $k_{1}, Y=\vec{\pi}-$ state detected 
by the detector pair $(b)$ on $k_{1}, Z=\vec{\pi}$ - state detected on the mode $k_{2}$. The experimental values are found in good agreement with the theoretical ones. The state probabilities related to the histogram variables $V H H$ and $H V H$, i.e. detected in coincidence with a $|H\rangle$ state realized on mode $k_{2}$, correspond precisely to the realization of the two interfering terms of the bipartite entangled state $|\Phi\rangle_{\text {out }}=2^{-1 / 2}\left(|H\rangle_{a}|V\rangle_{b}+|V\rangle_{a}|H\rangle_{b}\right)$ over the modes $a$ and $b$. However the existence of the contributions $\mathrm{VHH}$ and $\mathrm{HVH}$ alone is not a sufficient proof of the entanglement feature, since the above observation is also in agreement with a statistical mixture of $V H H$ and $H V H$. To demonstrate the coherent superposition of the two terms we further performed a polarization measurement in the $45^{\circ}$ basis on the modes $a$ and $b$ by rotating the half-wave plates $W P_{a}$ and $W P_{b}$ by $22.5^{\circ}$. In this basis $|\Phi\rangle_{o u t}$ is expressed as $2^{-1 / 2}\left(\left|+45^{\circ}\right\rangle_{a}\left|+45^{\circ}\right\rangle_{b}+\left|-45^{\circ}\right\rangle_{a}\left|-45^{\circ}\right\rangle_{b}\right)$ where $\left| \pm 45^{\circ}\right\rangle=2^{-1 / 2}(|H\rangle \pm|V\rangle)$. We measured the polarization correlation between the photons $a$ and $b$ with a four-coincidences scheme involving the detectors $\left(D_{T}, D_{2}, D_{a}, D_{b}\right)$. The correlation measurement was compared with the configuration in which there was no temporal overlap between the injected photon and the back reflected UV pump. In this case the two detected photons over the modes $a$ and $b$ have no correlation in the $45^{\circ}$ basis and hence there is the same probability for the photons to have the same or different polarization. By moving the mirror $M_{p}$ in order to continuously reach the temporal superposition, we should observe an increase of the coincidence counts by a factor $R=2$ for the position $Z=0$ (Fig. 3). Fitting the experimental data with a gaussian function, we estimate $R=1.68 \pm 0.07$. We note that the peak of Fig. 3 does not arise as an amplification process since the component $|H\rangle_{k 2}$ is not amplified (see Ref. [12,4]), instead it must be interpreted as a consequence of the mode coalescence of two photons with orthogonal polarization.

In conclusion we have experimentally demonstrated that the universal NOT gate process lies at the basis of any universal entangling device. The experiment enlightens the significance of the transformation Eq.(2) that contextually implements, in a unifying manner, the universal NOT gate, the universal optimal quantum cloning and the universal quantum entangler. Indeed the optimality and the universality of the entangling process is found 
to arise as a consequence of the same properties characterizing the cloning and the spinflipping processes [13]. Finally note that the optimal quantum entangler here realized in a more general NL context by a Optical Parametric Amplifier can also be implemented by a linear state-symmetrization procedure involving the simultaneous realization of the optimal quantum processor by a general modified quantum teleportation scheme $[14,11]$.

We thank Daniele Pelliccia for early collaboration in the experiment. This work has been supported by the FET European Network on Quantum Information and Communication (Contract IST-2000-29681: ATESIT), the Marie Curie Research and Training Network CONQUEST (Contract MRTN-CT-2003-505089) and by PRA-INFM 2002 (CLON). 


\section{REFERENCES}

[1] A. Peres: Quantum Theory: Concepts and Methods (Kluwer Academic, Dordrecht, 1993).

[2] W.K. Wootters and W.H. Zurek, Nature (London) 299, 802 (1982).

[3] V. Bužek and M. Hillery, Phys. Rev. A 54, 1844 (1996).

[4] A. Lamas-Linares et al., Science 296, 712 (2002)

[5] F. De Martini, D. Pelliccia and F. Sciarrino, Phys. Rev. Lett. 92, 067901 (2004): D. Pelliccia et al., Phys. Rev. A 68, 042306 (2003).

[6] V. Bužek, M. Hillery, and R.F. Werner, Phys. Rev. A 60, R2626 (1999).

[7] N. Gisin and S. Popescu, Phys. Rev. Lett. 83, 432 (1999).

[8] F. De Martini et al., Nature (London) 419, 815 (2002).

[9] J.S. Bell: Speakable and Unspeakable in Quantum Mechanics (Cambridge Univ. Press, Cambridge, 1987)

[10] L. Hardy, Phys. Rev. Lett. 71, 1665 (1993); D. Boschi, et al., ibidem 79, 2755 (1997).

[11] V. Bužek and M. Hillery, Phys. Rev. A 62, 022303 (2000); M. Hillery, V. Bužek, and M. Ziman, Phys. Rev. A 65, 022301 (2002).

[12] F. De Martini, Phys. Rev. Lett. 81, 2842 (1998).

[13] Note however that the no-cloning and the no spin-flipping theorems imply the impossibility of violating two entirely different QM axioms, namely the linearity and the complete positivity $(\mathrm{CP})$ of the corresponding maps. All CP maps are linear while the contrary is not generally true (K. Kraus, States, Effects and Operations (Spinger-Verlag, Berlin, 1983).

[14] M. Ricci, et al., Phys. Rev. Lett. 92, 047901 (2004); F. Sciarrino, et al., Phys. Lett. A 
323, 34 (2004); F. Sciarrino, et al., submitted to Phys. Rev. A.

\section{Figure Captions}

Figure.1. Schematic diagram of the self-injected Optimal Parametric Amplifier. The universal optimal quantum entangler is realized on the cloning $(\mathrm{C})$ channel (mode $k_{1}$ ). Micrometric adjustments of the coordinate $\mathbf{Z}$ of the UV mirror $M_{p}$ ensured the time superposition in the active NL crystal of the UV 140 femtosecond pump pulses and of the single photon pulse injected via back reflection by the fixed mirror $M$.

Figure.2. Probability distribution for the variables $X Y Z$ where $X, Y$ and $Z$ are the polarization $\vec{\pi}$-state detected, respectively, by the detector pair $(a)$ on the mode $k_{1}$, the detector pair $(b)$ on the mode $k_{1}$, and the detector pair (2) on the mode $k_{2}$. Each correlation data has been measured in a time of $2400 \mathrm{~s}$.

Figure.3. Coincidence counts $\left(D_{T}, D_{2}, D_{a}, D_{b}\right)$ versus the position $Z$ of the UV mirror $M_{p}$. The enhancement in the coincidence counts is a signature of the entanglement of the state $|\Phi\rangle_{\text {out }}$ 


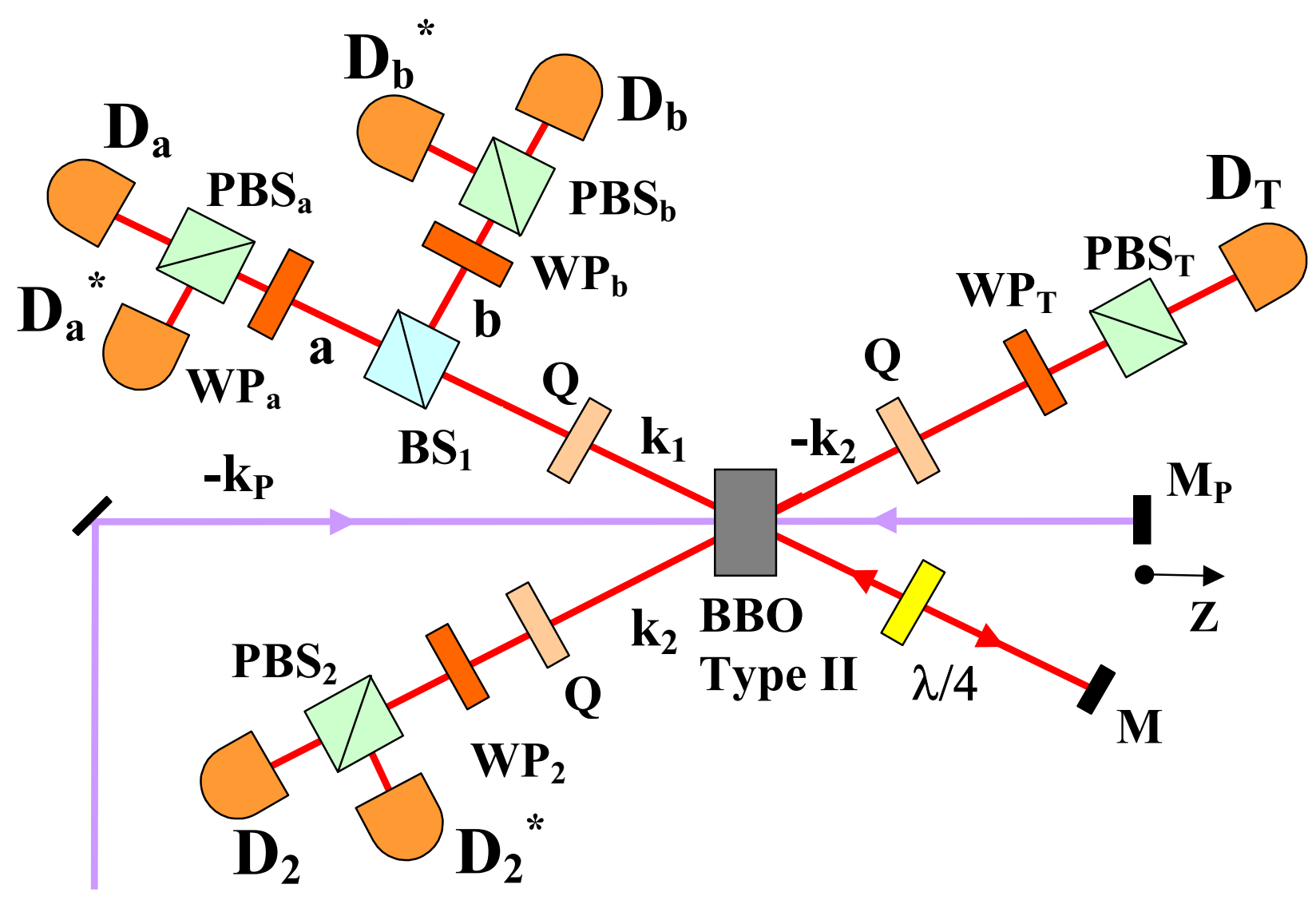




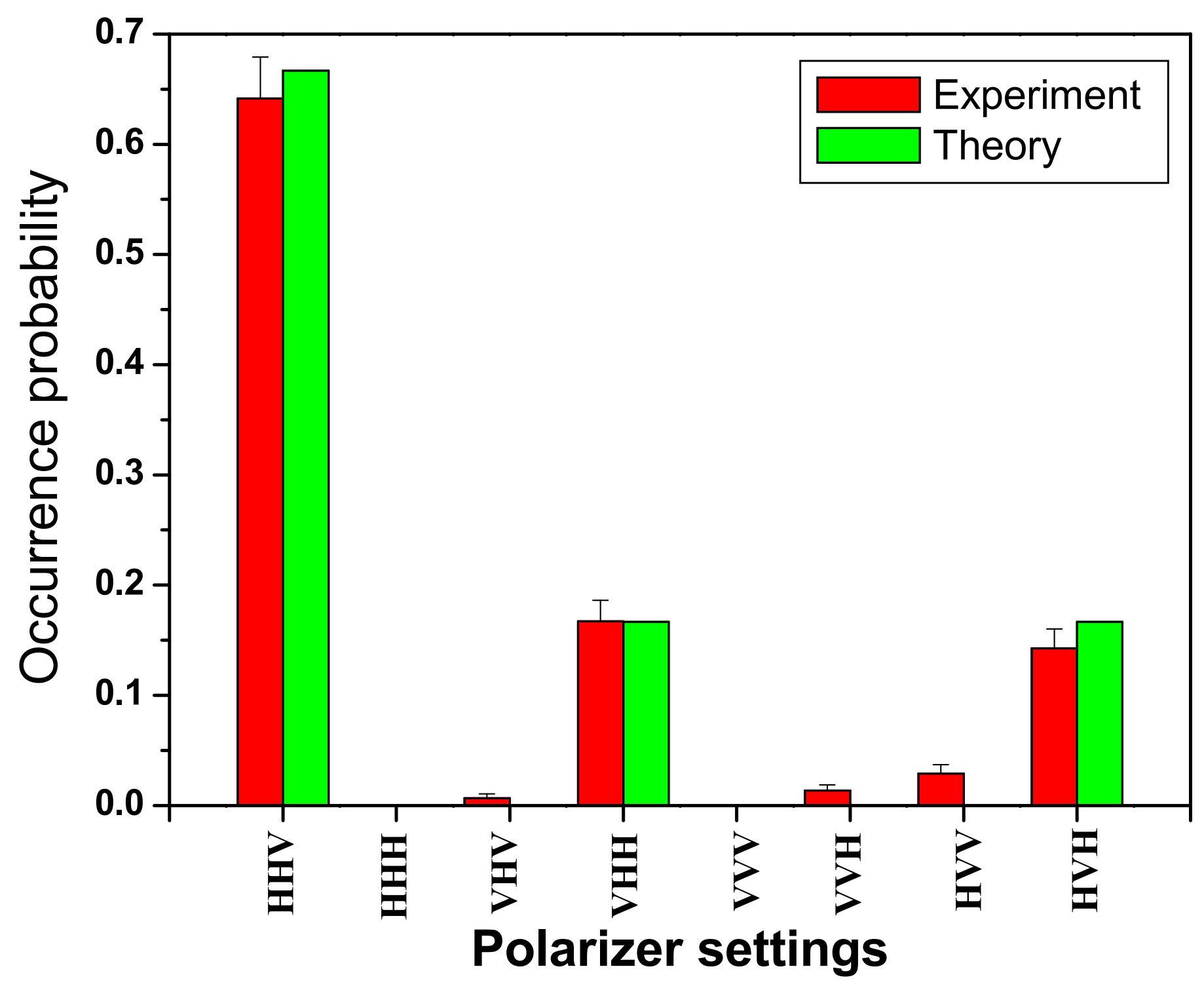




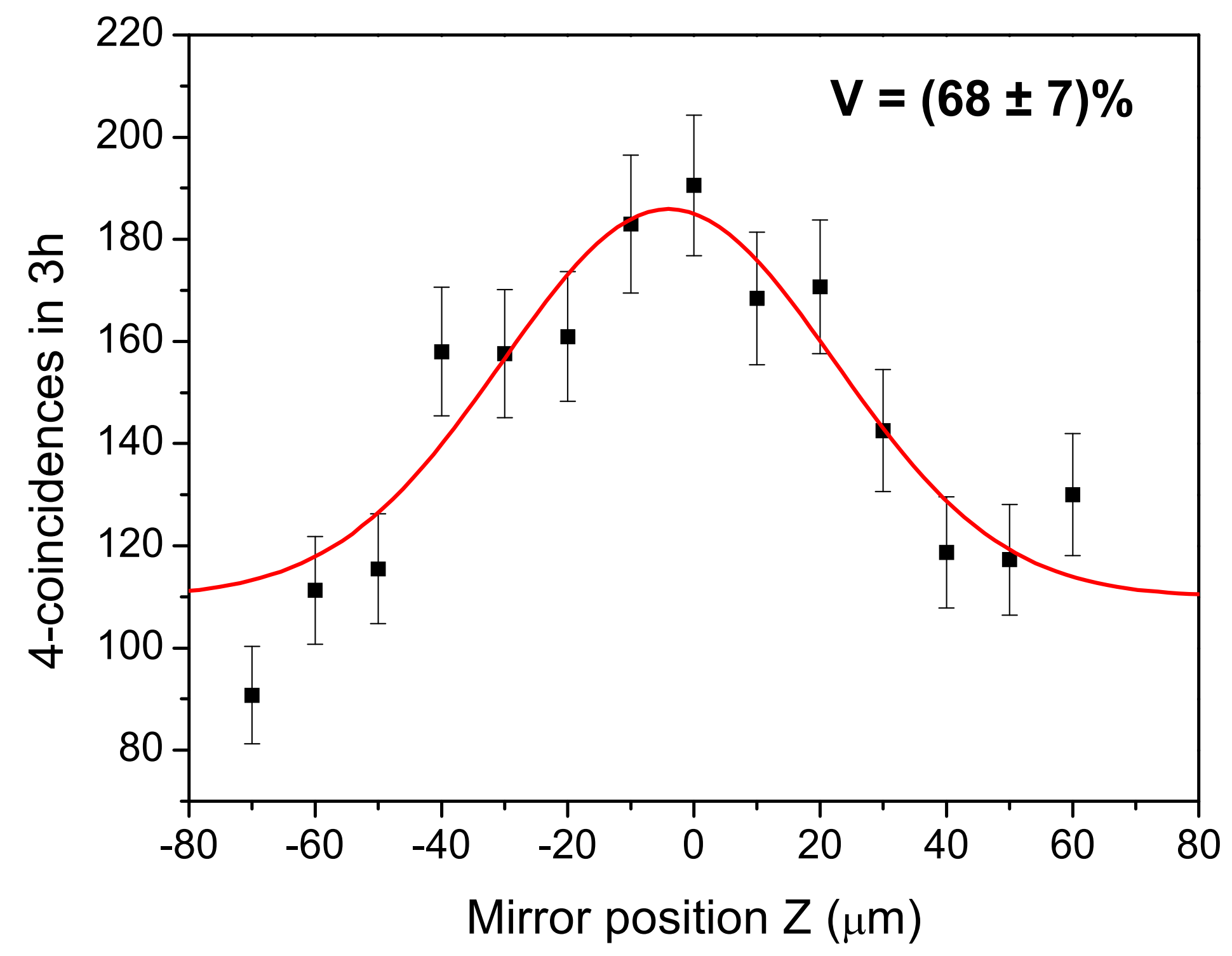

